\title{
EL BALNEARIO DE BELLÚS EN LOS SIGLOS XVIII Y XIX, A TRAVÉS DE LOS TRATADOS DE HIDROLOGÍA MÉDICA
}

\author{
Juan Antonio Micó Navarro \\ Instituto de Estudios Documentales e Históricos sobre la Ciencia. \\ Universitat de València C.S.I.C. Avda. Blasco Ibañez, 17. - 46010 Valencia \\ Amparo Soler Sáiz \\ Colaboradora del C.S.I.C. Instituto de Estudios Documentales e Históricos sobre la Ciencia. \\ Universitat de València C.S.I.C. Avda. Blasco Ibañez, 17. - 46010 Valencia
}

\section{RESUMEN}

El presente trabajo pretende dar a conocer la situación del balneario valenciano de Bellús, a través de los tratados de hidrología médica de los siglos XVIII y XIX que se conservan en la Biblioteca y Museo Historicomédicos de Valencia. Para ello se describe las condiciones arquitectónicas y técnicas del edificio, así como los diversos análisis de las aguas y su utilización terapéutica.

\section{SUMMARY}

The present work aims to explain the situation of the valencian bathing place in Bellús, through some treatises of medical hydrology from the 18th and 19th centuries which belong to the Library and Historico-medical Museum of Valencia. The architectural and technical conditions of the building are described, as well as the methods of water analysis and the therapeutical use of water.

\section{ANTECEDENTES HISTÓRICOS Y DESCRIPCIÓN DEL BALNEARIO EN EL SIGLO XIX.}

No hemos podido averiguar, a través de los textos que hemos consultado, la antigüedad de la utilización de las aguas de Bellús con una finalidad claramente curativa ${ }^{1}$. Aunque posiblemente fueran ya conocidas en época romana, la denominación que se

\footnotetext{
1 Para la realización del presente artículo hemos consultado los tratados de hidrología médica del siglo XVIII y XIX que se conservan en la Biblioteca y Museo Historicomédicos de Valencia, que citamos a lo largo de las siguientes notas. 


\title{
JUAN ANTONIO MICÓ NAVARRO Y AMPARO SOLER SÁIZ
}

da al manantial, conocido con la palabra árabe de "Alfama", que significa "baño caliente o aguas minerales" ${ }^{\prime 2}$, nos indica que en esta época debió tener ya una utilización médica o al menos que eran conocidas y utilizadas entre la población musulmana de Játiva.

No obstante, según afirma Benigno Villafranca en su documentada monografía sobre el balneario ${ }^{3}$, ya en el siglo XIX no se conservaban vestigios de construcciones romanas o medievales. Como veremos a través de los textos consultados, aunque ya en las últimas décadas del siglo XVIII encontramos referencias a la concurrencia de enfermos a este paraje, las primeras edificaciones balnearias fueron construídas a principios del siglo XIX.

Así, el testimonio más antiguo que hemos encontrado data de 1788. Pertenece a Francisco José de Lemos, quien en su obra titulada Virtudes medicinales de las aguas minerales de la Villavieja de Nules, y en un apéndice de la misma titulado "De las aguas minerales que hay más conocidas en el Reyno de Valencia"4, nos describe el manantial de Bellús en los siguientes términos:

\begin{abstract}
"Las aguas llamadas comunmente de Bellús están como á un quarto de hora del lugar de Guadacequies, pueblo inmediato a S. Felipe o Xátiva, y distante 10 leguas de Valencia. Esta agua es termal, pero su calor es muy moderado; de modo, que se bebe a todo uso sin molestia ni fastidio. Su manantial es copioso, y tiene proporción para tomar baños; pero con tanta incomodidad, que no hay ni aun disposición para defenderse de los calores del Sol mientras se está en el agua, ni tampoco para abrigarse los enfermos y libertarse de las injurias del ayre quando salen de ella, lo que es indispensable en todo baño de esta naturaleza, a fin de conservar el sudor, motivo por qué y por ser el parage muy propenso a tercianas, a causa de los arrozes que llegan hasta la misma fuente, son poco freqüentadas estas aguas, no obstante sus buenos efectos."
\end{abstract}

Aunque no es un tratado hidrológico, como el resto de obras consultadas, queremos resaltar la extrañeza que nos ha causado que Antonio José Cavanilles no cite las

\footnotetext{
2 El diccionario Alcover-Moll, nos da como origen etimológico de este toponimo la palabra árabe al-hamma , y compara este nombre con el castellano Alhama, que llevan distintas poblaciones conocidas también por sus aguas mineromedicinales. (Cf.: AlCOVER, A.; MOLL, F. B. (1985), Diccionari CatalàValencià-Balear, Palma de Mallorca, vol. I, p. 487).

3 Este folleto es una de las obras en que hemos basado nuestro trabajo. Benigno Villafranca y Alfaro fue médico-director, por oposición, del cuerpo facultativo de aguas y baños minerales. Destinado a Bellús en 1860, publicó cuatro años más tarde esta obrita con materiales que podemos considerar como una auténtica fuente documental. En él se queja constantemente tanto de las pésimas instalaciones del balneario como de su situación profesional, pero es un testimonio importante para conocer el estado higiénico-sanitario de este centro termal. (Cf.: VILLAFRANCA Y ALFARO, B. (1864), Monografía de las aguas salinas-termales de Bellús, Madrid, Imp. de Vicente y Lavajos, 99 p.).

4 Cf.: LEMOS, F. J. de (1788), Virtudes medicinales de las aguas minerales de la Villavieja de $\mathrm{Nu}$ les en el Reyno de Valencia, Valencia, Josef y Tomás de Orga, pp. 146-148.
} 


\section{EL BALNEARIO DE BELLÚS EN LOS SIGLOS XVIII Y XIX, TRAVÉS DE LOS TRATADOS DE...}

propiedades curativas de las aguas de Bellús en sus Observaciones sobre la historia natural... del Reyno de Valencia, publicadas en 1797, cuando sí lo hace de las de Villavieja en Castellón ${ }^{5}$ o las de Busot en Alicante 6 . No obstante nos puede servir como testimonio de las condiciones en las que se encontraban las instalaciones balnearias de nuestra Comunidad, confirmando la precariedad de los edificios, como ya nos decía Lemos. Cavanilles afirma:

"No hay comodidad alguna. El enfermo o los que le acompañan deben hacer de antemano las provisiones para subsistir. Es sobrado general el abandono que reyna en las fuentes saludables del Reyno: solo en Catí he visto una hospedería decente. La Font calenta entre Chestalgar y Chulilla, Toga, Navajas, Montanejos y la Vilavella, son sitios capaces y dignos de notables mejoras".

Si estos otros manantiales tenían una mala infraestructura, en el caso de Bellús no existía en absoluto. Respecto a esta población, Cavanilles sólo insiste, desde el punto de vista sanitario, en el tema del cultivo del arroz y sus miasmas que convierten la zona en un lugar de frecuentes brotes de tercianas ${ }^{7}$.

Fue a comienzos del siglo XIX cuando se construyó un edificio en Bellús para la atención de los enfermos. Veamos cómo lo describe Villafranca:

\footnotetext{
"... a principios de este siglo hubo de aumentar la concurrencia; los bañistas, en mayor número, no podrían servirse como desearían, se daría lugar á excesos y abusos de consideración, y un médico... llamado Climent, consiguió en 1803 a 1805 se erigiese el edificio que hoy existe con ligerísimas modificaciones. La mezquindad de este llega al punto de no poder alojar a persona medio regular" 8 .
}

Villafranca sigue quejándose del estado lamentable en que se encuentran los caminos que conducen a Bellús que califica de "intransitables" y que no exista en el pueblo un lugar apto para hospedar a la gente que va a tomar los baños, con un mínimo de condiciones. Por ello, comprende que el propietario de los mismos, el marqués de Bélgida, no invierta dinero en mejorar las instalaciones, pues la gente que acude no es, como ocurre en otros balnearios de España, de "aquella que deja productos al país y crédito a los baños." No obstante, durante la guerras carlistas que se desarrollaron por la provincia de Castellón, las cuales hicieron peligroso e inseguro acercarse a Benasal o a Villavieja, se desplazaron a Bellús muchos enfermos. Por eso se dio a esta plaza el carácter de planta en 1840.

5 Cf:: CAVANILlES, A. J. (1797), Observaciones sobre la historia natural, geografia, agricultura, población y frutos del Reyno de Valencia, Madrid, Imp. Real, vol. II, p. 247.

6 Idem.

7 Idem., vol. II, pp. 127, 128, 221.

8 Villafranca y Alfaro, B., Op. cit., p. 39. 


\section{JUAN ANTONIO MICÓ NAVARRO Y AMPARO SOLER SÁIZ}

En toda la primera mitad del siglo XIX, tan sólo se hizo una reforma siendo director de los baños el doctor Ildefonso Martínez Fernández, la cual describe Villafranca de la siguiente manera:

"consiguió que en lugar del techo a bovedilla de las balsas, que con la humedad y constante calor del agua se resquebrajaba y destruía, se pusiese una bóveda de piedra y yeso, pero no sin que antes se viniese abajo uno de los techos de las charcas o piscinas, que por fortuna no causó las desgracias que fácilmente podía haber ocasionado" 9 .

Este médico ganó la plaza por oposición en 1850, en sustitución de Victoriano Usera, quien la había ocupado desde 184710 .

Pedro María Rubio nos describe someramente las instalaciones de los baños en 1853 en su Tratado... de las fuentes minerales de España. Dice que:

"Estos baños consisten en cinco balsas, encerradas en un edificio que tiene 70 pies de largo, 18 de ancho y 25 de altura. Dichas balsas son bastante espaciosas para que entren á la vez varias personas; estan divididas para hombres y mujeres y ocupan todo el piso bajo. El piso alto del mencionado edificio tiene cuartos con una mala cocina."

"Pagan por cada baño los que son pobres 2 reales, cuyo precio cobraba en 1840 un labrador de Guadasequies, á quien se les tiene arrendados en 150 pesos anuales el Excmo. señor marqués de Bélgida."

"Los bañistas se alojan en las inmediatas poblaciones de Bellús y Guadasequies, de donde pasan diariamente a tomar los baños" 11 .

Pero la descripción más completa y fiable que hemos encontrado es la que nos proporciona, con amargura y frustración por el estado lamentable del establecimiento, su médico director Benigno Villafranca, y que corresponde a 1864:

"El edificio es un cuadrilongo de 26 metros de largo en la dirección de N. a S., cinco metros de ancho y siete de altura; es de cal y canto, y su aspecto exterior es el de un corral para encerrar ganados. En la fachada que mira a Poniente tiene siete agujeros, que llaman ventanas, cuatro escalones de yeso medio destruidos y una puertecita que da entrada al piso alto de la casa, el cual se compone de un largo y estrecho corredor transversal con seis habitaciones, tres a un lado y tres a otro; las dos primeras, tanto a la derecha como a la izquierda,

9 Idem., p. 40.

10 Según Rubio hubo otro médico-director de estos baños que ocupaba la plaza en 1838. (Cf.: RuBio, P. M. (1853), Tratado completo de las fuentes minerales de España, Madrid, Est. Tip. de R. R. de Rivera, p. 250). Despues de éste ocuparían la plaza tras ganar la correspondiente oposición: Victoriano Usera desde 1847; Ildefonso Martínez Fernández desde 1856; Benigno Villafranca desde 1859; Morales Gutiérrez desde 1877; Salvador desde 1878; Núñez desde 1884; Fernández Campá desde 1887; Courel desde 1891; Aleixandre desde 1892 y Salvador desde 1893. (Cf:: MARTíneZ REGUERA, L. (1897), Bibliografia hidrológico-médica española, Madrid, Sucesores de Rivadeneyra, vol. II, p. 878).

11 RUBIO, P. M., Op. cit., pp. 250 y 251. 


\section{EL BALNEARIO DE BELLÚS EN LOS SIGLOS XVIII Y XIX, TRAVÉS DE LOS TRATADOS DE..}

son destinadas para bañistas pobres, que no pueden por su enfermedad o por falta absoluta de recursos ir a alguno de los pueblecillos limítrofes; todas ellas son cuadradas, pequeñas, sin muebles de ninguna clase, con el techo de cañizo y abuhardillado, el pavimento de yeso, una puerta que apenas cierra, y una ventanilla a Poniente de pie y medio de alta por uno de ancha, sin cristal, como todas las de la casa. Al extremo izquierdo del pasillo trasversal está el despacho del médico-director, que forma una habitación algo mayor que las anteriores, pero más baja de techo por estar el pavimento levantado sobre las otras media vara, el techo de cañizo e inclinado, dos ventanas destartaladas, cuatro sillas de esparto y una mesita miserable y negra en un rincón, con un tintero de hoja de lata, esto es, un ajuar impropio de un médico que debe tener cierta autorización y carácter en un establecimiento de baños, y a quien es regular se le guarden consideraciones que tiene muy merecidas. En el extremo opuesto del corredor hay una cocina grande, pero mala."

"El piso bajo le ocupan una cuadra a la derecha de la puerta de entrada, tan mal arreglada como todo lo demás, y en ella hay un común montado al aire. En la fachada de Levante... se ven ocho ventanillas tocando al tejado, de las cuales dos están tapiadas y las demás dan luz al pasillo trasversal mencionado y cocina, por debajo estan las cinco puertas que dan entrada a los baños. Los dos primeros se destinan a mujeres, los dos segundos a hombres, y el último se llama de pobres, aun cuando el estado en que se le tiene hace que se use poco. Las balsas son casi iguales y ocupan las dos terceras partes de la habitación, tienen dos metros y medio de ancho por dos de largo, están excavadas en la misma roca y por su fondo sale sin interrupción el agua que las llena; las cubre una bóveda bien hecha, aunque baja. Las primeras son lisas y poco hondas, la tercera lo es más, y la cuarta, que llaman enfáticamente Baño del Marqués, tiene en su centro un metro de fondo, es muy desigual, la peña está cortada a pico, y los pies suelen sentir con frecuencia la falta de lisura en su superficie; la quinta y última recibe el agua por un gran caño que la atraviesa en la pared del S. Todas tienen su puerta al campo y una ventanita sin cristal ni encerado a Poniente: un banco de yeso y una mala percha completan el atalaje de las piezas de baños, en los que a fuer de piscinas se bañan seis, ocho y aun más personas"12.

También se queja Villafranca de la total falta en Bellús de equipamiento médico y técnico que existe en cualquier balneario mínimamente dotado como son las duchas de vapor, pilas individuales para que los enfermos tomen el baño, así como:

"carecer de una habitación que les preceda y estar los bañistas obligados a salir del baño al aire libre, y precisamente por el lado donde soplan vientos más frecuentes y frescos... se echan de menos esas cosas que hasta en los humildes baños de Manzanera se encuentran, sillas, esteras, mesas, y cuanto se necesita para vestirse y desnudarse"13.

En 1876, Anastasio García López, en su Guía del Bañista se queja nuevamente del lamentable estado de las instalaciones termales de Bellús, de las que dice que

12 Villafranca y Alfaro, B., Op. cit., pp. 41 y 42.

13 Idem., p. 43. 


\section{JUAN ANTONIO MICÓ NAVARRO Y AMPARO SOLER SÁIZ}

están "en la infancia de esta industria"14 repitiendo en pocas líneas las quejas de Villafranca.

Unos años más tarde, en el Anuario oficial de las aguas minerales de España, editado en 1883, y entre cuyos redactores se encontraban Anastasio García López, Benigno Villafranca y Leopoldo Martínez Reguera, encontramos dos datos a resaltar. En primer lugar, el nombre del facultativo que se encontraba entonces al frente del balneario, Juan Bautista Bernabeu, al que dan el calificativo no de médico-director de la plaza sino de "interino"; y en segundo lugar, que el marqués de Bélgida, que tanta desidia había demostrado por la mejora de las instalaciones balnearias, ya no era el propietario, sino que lo había vendido a Juan Bautista Mompó ${ }^{15}$. Esto debió ocurrir hacía la década de los ochenta, pues el Anuario nos dice que la instalación es aún mala, aunque "su actual propietario empieza a efectuar las obras necesarias para la construcción de un buen estableciemiento"16.

A Mompó se deben la introducción de dos grandes mejoras: la primera, el haber encargado el análisis químico de las aguas al catedrático de Terapéutica de la Universidad de Valencia Vicente Peset, persona de reconocido prestigio científico, que emitió su informe, del cual hablaremos más adelante, el 14 de enero de 1899; la segunda, fue la transformación del edificio balneario de una construcción rudimentaria, antihigiénica y lamentable, como hemos visto por los testimonios aportados, en que la mantenían los marqueses de Bélgida, residentes en Madrid y desvinculados afectivamente hacía tiempo de sus propiedades valencianas, en una construcción arquitectónica moderna y dotada de los servicios necesarios no sólo para la asistencia médica al enfermo, sino para su entretenimiento lúdico. Esta nueva construcción está minuciosamente descrita en un folleto titulado Balneario de Bellús, editado en Valencia en 1901. Creemos de interés reproducirla para contrastarla con la realizada unos años antes con Villafranca. Dice así:

"El edificio, construido de nueva planta, en el que existe Oratorio ó Capilla pública, reune condiciones inmejorables, tanto por su capacidad como por la distribución que se ha dado a sus espaciosas y bien amuebladas habitaciones para que los concurrentes al Balneario puedan disfrutar de toda clase de comodidades."

14 García López, A. (1875), Hidrología Médica, Salamanca, Imp. Sebastián Cerezo, vol. II, pp. 186 y 187; GARCíA LÓPEZ, A. (1876), Guía del Bañista o Compendio de hidrología médica, $2^{\mathrm{a}}$ ed., Salamanca, Imp. Sebastián Cerezo, pp. 172 y 173.

15 Debió ser el septimo marqués Iñigo Alvarez de las Asturias Bohorquez, que sucedió a su padre en la posesión del título y mayorazgo en 1852, quien debió enajenar los baños a Juan Bautista Mompó hacia los años ochenta del pasado siglo, pues éste comenzó por estas fechas la reforma del edificio (Cf.: JORNET PERALES, M. (1932), Bélgida y su término municipal, Valencia, Hijos de Vives Mora, p. 448). y 366.

16 ANUARIO oficial de las aguas minerales de España, (1883), Madrid, M. Minuesa, vol. II, pp. 365 


\section{EL BALNEARIO DE BELLÚS EN LOS SIGLOS XVIII Y XIX, TRAVÉS DE LOS TRATADOS DE...}

\footnotetext{
"Se ha establecido una Estación Telefónica enlazada con la Red Telegráfica del Estado, para que los señores bañistas puedan utilizar durante la temporada este importante medio de rápida comunicación".

"Existe una completa instalación de Pararrayos en el Edificio y de Timbres Eléctricos en todas las habitaciones."

"El Salón de Recreo ha quedado definitivamente establecido con las mejoras necesarias, a fin de que los señores bañistas puedan disfrutar, con todas las comodidades, de tan indispensable departamento."

"Los Comedores, tanto de $1^{\text {a }}$ como el de $2^{\mathrm{a}}$, son espaciosos, y en el primero se han establecido mesas pequeñas para quienes deseen comer por separado, con un pequeño aumento sobre el precio del cubierto."

"Se han construido un Paso Cubierto entre la Fonda y el Establecimiento de Hidroterapia, y un nuevo Pabellón de Retretes Inodoros, y se han instalado Fuentes en todos los pisos del Balnerario"17.
}

Así mismo nos da una lista de precios de los baños por piscina, en pila o ducha y del alquiler de sábanas y toallas. También los de las comidas, que oscilaban entre dos y cuatro pesetas pensión completa.

Mompó solucionó otro de los grandes inconvenientes de este establecimiento termal, que eran las carreteras y accesos a Bellús, de los que Villafranca se quejaba definiéndolas como "intransitables caminos que a él conducen"18. Este nuevo propietario estableció un servicio de carruajes para los asistentes al balneario, que los recogía al bajar del tren de Játiva y que podían ser alquilados por los residentes en el balneario para realizar excursiones por los lugares cercanos. Era una nueva concepción comercial y moderna, diametralmente opuesta a la seguida por los anteriores dueños, los marqueses de Bélgida, que lo consideraban una simple fuente de ingresos económicos, como si de un campo de olivos se tratase.

Pero aparte de residir en el balneario como en un hotel, éste disponía de:

"habitaciones espaciosas, independientes y ventiladas, con camas, ropa para las mismas, vajilla y cocina propia, para que las familias que lo deseen puedan confeccionarse la comida por su cuenta"19.

Para ello el balneario tenía una tienda bien abastecida de comestibles, vino, petróleo, jabón y bujías "a precios limitadísimos".

La temporada de baños en el siglo XVIII duraba desde últimos de mayo a San Juan, o sea el 24 de junio, porque, como dice Lemos :"después empiezan las tercia-

17 BALNEARIO de Bellús, (1901),Valencia, Imp. Doménech, $4 \mathrm{f}$.

18 "Bellús está casi incomunicado. El camino de Játiva es de herradura, pésimo y algunas veces espuesto; los que parten de Beniganim, la Puebla y Montaberner, son peores, y la única esperanza es la conclusión de la carretera de Alicante." (Cf.: VillafranCA Y AlFARO, B., Op. cit., p. 98).

19 Cf.: BALNEARIO de Bellús, f. 2 r. 


\section{JUAN ANTONIO MICÓ NAVARRO Y AMPARO SOLER SÁIZ}

nas por todo aquel parage"20. Villafranca afirmaba que "llegaban las charcas hasta la misma casa balnearia"21. Pero al desaparecer este inconveniente, producido por el encharcamiento de los campos para el cultivo del arroz, cuya producción fue prohibida en 1823, la temporada se alargaría. Así Raimundo Monasterio, en 1850 nos informaba que había dos temporadas de baños. La primera comprendía del 15 de abril al 15 de julio y la segunda del 15 de septiembre a finales de octubre ${ }^{22}$. Esta división se vería alterada hacia los últimos años de la centuria y en el folleto editado en 1901, tras la construcción del nuevo edificio al que hemos hecho referencia, vemos que la primera temporada comprendía del 1 de mayo al 30 de junio y la segunda del 15 de agosto al 31 de octubre 23 .

\section{ANÁLISIS DE LAS AGUAS.}

La primera descripción de la composición de las aguas de Bellús que hemos encontrado es la que nos proporciona Lemos en su obra. Dice que:

\footnotetext{
"Ellas son muy claras, carecen de olor y tienen un sabor nada ingrato, solo que al tragarlas se conoce que son algo gruesas, pero frías ya no se advierte tanto. No dexan vestigios de sustancia conocida en su corriente, ni remansos, y lavándose con ellas denotan que son de naturaleza saponácea, pues dexan las manos muy suaves, y la piel parece que se vuelve más blanca quando se está dentro del baño"24.
}

No será hasta mediados del siglo XIX cuando encontremos un primer acercamiento al análisis de los componentes de las mismas. Así en 1850, Monasterio las describe con un temperatura de:

" $21^{\circ} \mathrm{R}$ : estan mineralizadas, aunque no sabemos en qué cantidad, por las sustancias siguientes: cloruros y sulfatos sódico y magnésico, carbonato bicálcico y bimagnésico"25.

Tres años más tarde, en 1853, Pedro María Rubio nos habla de los análisis llevados a cabo por Serafín García en 1840, por un médico de Játiva llamado Todolí y por Victoriano Usera. Esta es la composición que nos ofrece, siguiendo un procedimiento aconsejado por Berzelius. Según esta curiosa descripción que cuantifica en "granos",

20 Cf..: LEMOS, F. J., Op. cit., p. 148.

$21 C f$ :: VillafRANCA Y ALFARO, B., Op. cit., p. 40.

22 Cf.: MONASTERIO Y CORREA, R. Op. cit., p. 323.

${ }^{23}$ Cf.: BALNEARIO..., f. 1 r.

24 Cf.: LEMOS, F. J., Op. cit., p. 147.

25 Cf.: MONASTERIO y CORREA, R., Op. cit., pp. 322 y 323. 
el agua de Bellús contenía: aire atmosférico, cloruros sódico y magnésico, sulfato magnésico y sódico, subcarbonato magnésico y cálcico y ácido silicílico ${ }^{26}$. Este mismo análisis fue reproducido por el Dictionnaire général des eaux minérales et d'hydrologie médicale, impreso en París en 1860, que al describir las propiedades de las aguas de Bellús, copia integramente el análisis realizado por Victoriano Usera, a quien citan, añadiendo las equivalencias de los "granos" en gramos ${ }^{27}$.

En 1875 Anastasio García López reproduce el análisis realizado por Usera, pero añadiendo a los componentes químicos detectados por aquel el ázoe, el óxido férrico y el sílice, y dando la medición de los gases en centímetros cúbicos y los minerales en gramos. Una prueba de lo rudimentario de estos análisis es la indicación de la existencia de "indicios" de sílice, del cual no hace una cuantificación ${ }^{28}$.

Así vemos cómo se van completando los distintos análisis químicos a medida que avanza el siglo, pues la aplicación de los conocimientos químicos a la medicina no será un hecho cotidiano hasta la introducción de la denominada "medicina de laboratorio" en las últimas décadas del siglo XIX. Es muy ilustrativa a este respecto la opinión de Benigno Villafranca sobre el particular cuando afirma:

\begin{abstract}
"Al echar mano de los reactivos químicos para arrancar á las aguas de Bellús el secreto de su composición, sentía una gran curiosidad y no menor desaliento: gran curiosidad porque había visto y oido algunos análisis hechos por profesores instruidos, y que se diferenciaban en gran manera; así por ejemplo, en 1838 un médico de Carcagente notó en ellos un debil olor sulfuroso y su sabor astringente de tinta bien marcado... el Sr. Clemencín, en 1840, halló en la misma cantidad de vehículo 14`3/5 grs. de sales, y el señor Usera 16`99 grs., con análogos componentes, en mil partes de agua: posteriormente alguno las ha comparado a las de Panticosa y las ha llamado azoadas; otros, carbónicas..." 29.
\end{abstract}

Villafranca realizó el análisis ayudado por su amigo Yañez, que poseía, según él, "clarísimos conocimientos y práctica en estudios químicos" llegando a detectar en la composición del agua dos tipos de compuestos: los gases y las sustancias fijas. En los primeros cita el aire atmosférico con exceso de ázoe y falta de oxígeno, y en los

26 Cf:: RUBIO, P. M., Op. cit., p. 250.

27 Cf.: DICTIONNAIRE général des eaux minérales et d'hydrologie médicale, (1860), Paris, J. B. Baillière et fils, vol. I, p. 242.

Esta obra escrita por Durand-Fardel, Eugène Le Bret, Lefort y Jules François, da curiosamente una noticia que nos ha llamado la atención, pues al hablar de los baños de Bellús dice: "On y trouve des ruines romaines et arabes", cuando ni Lemos, ni Cavanilles, ni Villafranca, que conocían el terreno a la perfección citan estas ruinas. Desconocemos de dónde pudieron obtener esta información los médicos franceses, a no ser el nombre árabe del nacimiento termal, que hace mención, como ya indicamos, a su utilización terapéutica en época musulmana.

$28 C f .:$ GARCíA LÓPEZ, A., Hidrología médica, vol. II, p. 186.

$29 C f$.: VillafRANCA YAlfaro, B., Op. cit., p. 47. 


\section{JUAN ANTONIO MICÓ.NAVARRO Y AMPARO SOLER SÁIZ}

segundos sulfato de calcio, magnesio y potasio; cloruro sódico, óxido férrico, sílice y agua pura. Todos estos elementos, que vienen a coincidir con los anteriores análisis químicos de las aguas, los expresa en gramos. Por último define estas aguas como "termales y salinas de la clasificación de Chenu, de composición muy débil; pertenecen a las sulfatado-cloruradas ( $1^{\mathrm{a}}$ clase) en la clasificación de Herpin; a las salinas sulfatadas de Henry; a las sulfatadas mistas de Durand-Fardel, y a las salinas mistas de cálcico-magnesianas de Petrequin y Socquet» ${ }^{30}$. Con estas definiciones podemos apreciar el auténtico "bosque" de clasificaciones de aguas termales en el que se movía la hidrología médica del siglo XIX. No es nuestra intención, dada la extensión del presente trabajo, entrar a explicar las diferentes técnicas en que se basaban estos análisis, sino hacer una somera descripción que permita a los interesados en el tema un acercamiento más detenido.

Para finalizar este apartado queremos hacer referencia al análisis químico de estas aguas que, por encargo de Juan Antonio Mompó, realizó el catedrático de Terapéutica de nuestra Facultad de medicina Vicente Peset Cervera el 14 de enero de 1899. Este profesor, que instaló en 1888 uno de los primeros laboratorios de análisis químicos que hubo en Valencia ${ }^{31}$, encontró los siguientes elementos: por una parte gases disueltos que comprendían distintas proporciones de oxígeno, nitrógeno y ácido carbónico; por otra, carbonatos de cal, sosa, magnesia y hierro, silicato de sosa y nitrato amónico. Define las aguas como acrotermas, bicarbonatadas y litínicas "lo que explica su ya antigua fama en los procesos reumáticos; pero conviene emplearla además en los trastornos digestivos y litiásicos" ${ }^{\prime \prime 2}$.

Como podemos comprobar este análisis es mucho más preciso en cuanto a la descripción de los componentes minerales de estas aguas, indicando las cantidades que se han encontrado de cada elemento en granos por litro de agua y sus equivalencias, en algunas de ellas, en bicarbonato o en centímetros cúbicos. Constituye ya la aplicación sistemática de los conocimientos químicos al estudio de la terapéutica médica.

\section{UTILIDAD TERAPÉUTICA.}

Hemos de remontarnos nuevamente al siglo XVIII, y en concreto a la obra de Lemos, para ver a qué tipo de enfermedades se aplicaban las aguas de Bellús como remedio curativo. Este las recomendaba para los :

\footnotetext{
30 Idem., p. 55.

31 Además de las aguas de Bellús pueden citarse sus análisis de las de la Font de Ensegures de Benasal y Villavieja de Nules en Castellón, las de Molinell o Nuestra Señora del Orito en Alicante o las de Cofrentes en Valencia. (Cf.: Micó NAVARro, J.A. (1994), "Vicente Peset Cervera (1855-1945), catedrático de Terapéutica y autor de las primeras radiografías valencianas", Médicos, 44, 9-12).

32 Cf.: BALNEARIO de Bellús... f. 2 v.
} 


\title{
EL BALNEARIO DE BELLÚS EN LOS SIGLOS XVIII Y XIX, TRAVÉS DE LOS TRATADOS DE...
}

\begin{abstract}
"accidentes apopléticos, siendo muchos los que con su riego han logrado el poderse manejar al tercer dia de tomarlas... Lo mismo han experimentado en los afectos reumáticos y gotosos, siempre que los dolores no han sido complicados con la lue-venérea, porque entonces salen del baño peores que entraron... Se han visto provechosos en la hipocondría, melancolía, pasión histérica, supresión de meses, fluxos blancos y demás achaques que tienen por causa una sangre espesa, viscosa y sin acción en el sólido para empujarla, por demasiada rigidez ó por defecto de contracción. Ellas curan perfectamente todo afecto cutáneo, como sarna, tiña, humor salado, empeines etc., resuelven las inchazones edematosas, y conducen a cicatrizar toda llaga antigua, que por razón de hinchazón en la parte ó abundancia de humores gruesos y viscosos sumamente adheridos á sus partes no pueden llegar a este estado" 33 .
\end{abstract}

Vemos en esta descripción una mentalidad basada en los conceptos hipocráticogalénicos de la medicina clásica, con referencias a los "humores" y "sangre espesa". Para contrastar vamos a reproducir las enfermedades para las que se consideraba adecuada en 1864, puesto que las otras obras consultadas coinciden con Benigno Villafranca, quien al haber sido médico-director del balneario, consideramos como fuente de mayor credibilidad. Este nos dice:

\footnotetext{
"se ha visto que producen buenos resultados en los reumatismos articulares y musculares sin lesiones orgánicas, en las que predomina el dolor hasta el punto de estar los enfermos imposibilitados parcial ó completamente del movimiento; en las neuralgias, señaladamente de índole reumática y las del estómago; en las enfermedades de la matriz, infarto, metritis crónicas, leucorrea, amenorrea e histerismo, y por último en muchos traumatismos. A pesar de que estos son los casos principales, si tuviéramos medios hidroterápicos completos, se podrían extender a muchos otros de parálisis, de alteraciones discrásicas, de enfermedades diatésicas rebeldes, que ahora no sufren modificación"134.
}

Villafranca desaconseja, por último, el tomar los baños de Bellús a las personas que tienen un proceso febril mientras dure éste, así como a aquellos que han padecido hemorragias cerebrales, cosa que contradice las indicaciones de Lemos. Así mismo las desaconseja en todos aquellos casos en que el enfermo padece lesiones orgánicas de estómago, hígado o cualquier otro órgano interno.

Nuevamente en el folleto editado en 1901 y basado en el análisis de Peset, se produce una contradicción con usos anteriores. Así, mientras Villafranca las desaconseja para lesiones del estómago e hígado, en este nuevo folleto de finales del XIX y comienzos del XX se aconsejan:

"tanto en los trastornos digestivos, que constituyen ciertas dispepsias anaclorhídricas e hipoclorhídricas, perezosas o bradipépsicas, y en varios afectos biliares o hepáticos e intestinales, como en los comunes amortiguamientos de la nutrición; también si se traducen por

33 Cf:: LEMOS, F.J., Op. cit., pp. 147 y 148.

$34 C f .:$ VillafRANCA YAlfaro, B., Op. cit., pp. 83 y 84. 
JUAN ANTONIO MICÓ NAVARRO Y AMPARO SOLER SÁIZ

\begin{abstract}
diabetes, polisarcia u oxaluria, y muy especialmente en el artritismo, desde sus manifestaciones profundas, catarros, gota, reuma y neurastenia, hasta las más externas, constituidas por ciertas dermatosis pruriginosas, litiasis o cálculos urinarios."

"Asimismo son muy especiales en el escrofulismo, anemia y clorosis, debilidad general, linfatismo y enfermedades del sistema nervioso. No tienen rival en las amenorreas y metrítis crónicas, en cuyos padecimientos se vienen observando desde antiguo múltiples y maravillosas curaciones" ${ }^{135}$.
\end{abstract}

Así pues, vemos cómo, pese a contradecirse unos textos con otros en el uso terapeutico de estas aguas, todos coinciden en su utilización en los procesos reumáticos e inflamatorios.

Esta nueva etapa, comenzada a fines del siglo XIX, conoció un sostenido auge a lo largo de la primera mitad del siglo XX, hasta que el abandono progresivo, por parte de la medicina científica académica, de la utilización terapéutica de las aguas minero-medicinales, llevó a muchos de estos establecimientos a su cierre parcial o definitivo.

Actualmente ha comenzado una recuperación del termalismo que deseamos devuelva a estos balnearios, como el de Bellús, su antiguo esplendor como lugar de curación y de reunión social. Sería beneficioso no sólo para nuestra salud, sino para la economía y el turismo de nuestras poblaciones de interior.

35 Cf.: BALNEARIO de Bellús... f. 1 v. 